\title{
Synthesis of a Polyimide Porous Porphyrin Polymer for Selective $\mathrm{CO}_{2}$ Capture
}

\author{
Venkata S. Pavan K. Neti, ${ }^{1}$ Jun Wang, ${ }^{2}$ Shuguang Deng, ${ }^{2}$ and Luis Echegoyen' \\ ${ }^{1}$ Department of Chemistry, University of Texas at El Paso, El Paso, TX 79968, USA \\ ${ }^{2}$ Department of Chemical Engineering, New Mexico State University, Las Cruces, NM 88003, USA \\ Correspondence should be addressed to Venkata S. Pavan K. Neti; u0982806@utah.edu and Luis Echegoyen; echegoyen@utep.edu
}

Received 3 December 2014; Revised 28 March 2015; Accepted 9 April 2015

Academic Editor: Ewa Schab-Balcerzak

Copyright ( 2015 Venkata S. Pavan K. Neti et al. This is an open access article distributed under the Creative Commons Attribution License, which permits unrestricted use, distribution, and reproduction in any medium, provided the original work is properly cited.

A new microporous porphyrin-based imide linked polymer, PyP, was synthesized in an $m$-cresol/isoquinoline mixture between a porphyrin and a naphthalene tetra-carboxylic dianhydride. The resultant amorphous polymer demonstrates a moderate surface area $\left(\mathrm{S}_{B E T}=428 \mathrm{~m}^{2} \mathrm{~g}^{-1}\right)$ and $\mathrm{CO}_{2}$ adsorption ability $(3.5 \mathrm{wt} \%)$ and $\mathrm{CO}_{2} / \mathrm{CH}_{4}$ selectivity of 5.6 at $273 \mathrm{~K} / 1$ bar.

\section{Introduction}

Polyimides (PIs) have been mainly investigated as lightweight and flexible materials which are resistant to heat and chemicals. PIs are usually prepared by the reaction between a dianhydride and a diamine and the resulting polymers possess high tensile strength and can be used as flame retardants [1]. Porphyrins are rigid and symmetric macrocycles suitable for making porous organic frameworks such as covalent organic frameworks (COFs) [2-4], metal organic frameworks [5] (MOFs), and porous organic polymers (POPs) [6]. Porphyrins are ideal candidates for nanoporous materials due to their light harvesting abilities and directionality, as illustrated by recent examples of their use within COFs and MOFs. Recently, POPs were shown to be an attractive alternative to MOFs due to their excellent thermal and chemical stabilities and great mechanical strength and because they can be easily synthesized from common organic reactions such as Suzuki and Sonogashira cross-coupling reactions, Yamamoto polymerization, and alkene and alkyne metathesis reactions [6]. POPs obtained by covalent bond formation instead of metal coordination MOFs exhibit better chemical and hydrothermal stabilities. The pore dimension of these porous organic polymers can be controlled by the appropriate choice of organic linkers. Over the past decade POPs and polymers of intrinsic microporosity (PIMs) [7, 8] and other conjugated microporous polymers (CMPs) have been developed using different chemical reactions. We have demonstrated that the use of COFs and POPs containing porphyrins connected by rigid imine bonds exhibits relatively high surface areas and $\mathrm{CO}_{2}$ capture [9-12]. The imide linkages of PIs are similar to those of other nitrogen containing frameworks such as zeolitic imidazolate frameworks (ZIFs) [13] and imine and benzimidazole linked frameworks [14-16]. The selective $\mathrm{CO}_{2}$ adsorption in these frameworks over $\mathrm{CH}_{4}$ or $\mathrm{N}_{2}$ is believed to arise as a consequence of $\mathrm{CO}_{2}$-framework interactions through $\mathrm{R}-\mathrm{N}\left(\delta^{-}\right)-\mathrm{C}\left(\delta^{+}\right) \mathrm{O}_{2}$ [17]. Porous aromatic polyimides were shown to possess high surface areas and selective $\mathrm{CO}_{2}$ capture properties; however, there are only a few reports to date $[16,18,19]$ and to the best of our knowledge $\mathrm{CO}_{2}$ capture properties of a porphyrin-based polyimide system have not been reported.

Here we report the synthesis and gas adsorption properties of a porphyrin-based polyimide framework containing meso-tetra(4-aminophenyl)porphine $\mathrm{Cu}$ (II) (1) and 1,4,5, 8-naphthalenetetracarboxylic dianhydride (2) (Scheme 1). The PyP was synthesized by the condensation reaction between meso-tetra(4-aminophenyl)porphine $\mathrm{Cu}(\mathrm{II})$ and naphthalenetetracarboxylic dianhydride using isoquinoline as a base and $m$-cresol as a solvent (see ESI). This polycondensation reaction allows the preparation of an inherently porous polymer which was isolated in 64\% yield as a purple powder. After repeated rinse with DMF, water, THF, acetone, and methanol, the polymer was purified by Soxhlet extraction 


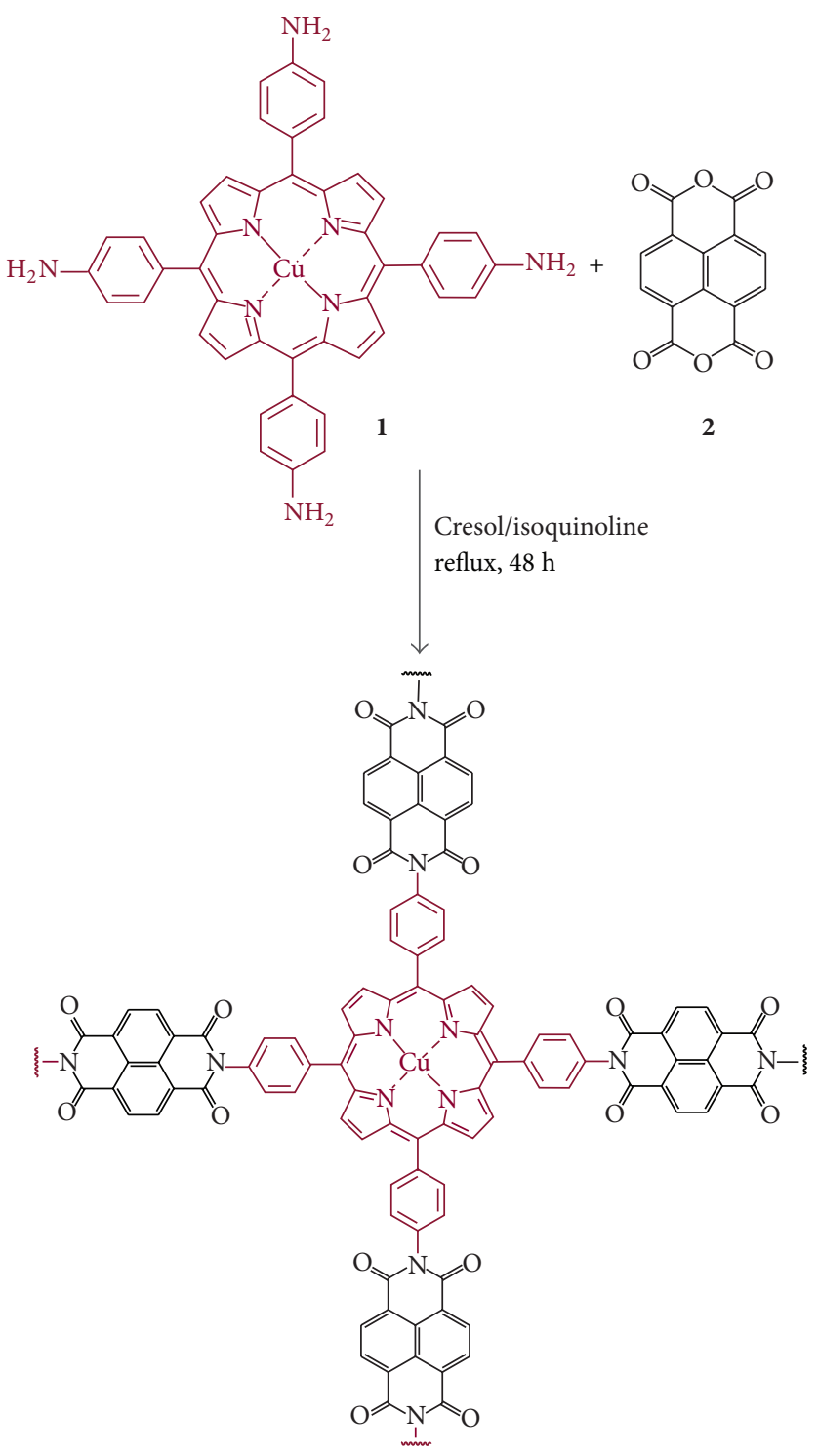

Scheme 1: Synthesis of PyP from meso-tetra(4-aminophenyl)porphine $\mathrm{Cu}(\mathrm{II})$ and 1,4,5,8-naphthalenetetracarboxylic dianhydride.

for $12 \mathrm{~h}$ with dichloromethane as solvent, to remove any trapped monomers, and then dried under vacuum. The PyP exhibits $\mathrm{CO}_{2}$ adsorption capacity of $3.5 \mathrm{wt} \%$ and $\mathrm{CH}_{4}$ capacity of $0.32 \mathrm{wt} \%$ at $273 \mathrm{~K}, 1 \mathrm{bar}$, and an adsorption capacity of $0.4 \mathrm{wt} \%$ for $\mathrm{H}_{2}$ at $77 \mathrm{~K} / 1$ bar. The chemical connectivity and composition of the PyP were characterized by several analytical methods such as infrared spectroscopy, solidstate ${ }^{13} \mathrm{C} C P-M A S ~ N M R$, surface area measurements, solidstate UV-Vis spectroscopy, powder $\mathrm{X}$-ray diffraction, and thermogravimetric and elemental analyses.

\section{Experimental Procedures}

All chemicals and solvents were purchased from SigmaAldrich. Fourier transform infrared (FT-IR) spectra were recorded on a Perkin-Elmer Spectrum One infrared spectrometer (ATR). Field-emission scanning electron microscopy (FE-SEM) was performed on a Hitachi S-4800 fitted with an EDAX energy-dispersive spectrometry system by adhering sample on a sampling platform. Matrix-assisted laser desorption ionization time-of-flight mass (MALDITOF MS) spectra were recorded on Bruker benchtop microflex model using matrix trihydroxyanthracene. In order to determine pore textural properties including the specific Brunauer-Emmett-Teller (BET) surface area, pore volume and pore size distribution, nitrogen adsorption, and desorption isotherm on free-flowing amorphous PyP sample at $77 \mathrm{~K}$ were measured in ASAP-2020 adsorption apparatus (Micromeritics). The as-synthesized samples were degassed in situ at $160^{\circ} \mathrm{C}$ with a heating rate of $3^{\circ} \mathrm{C} / \mathrm{min}$ under a vacuum $(0.0001 \mathrm{mmHg})$ for $12 \mathrm{~h}$ before nitrogen adsorption measurements in order to ensure that the microchannels in the structure were guest-free. The Brunauer-EmmettTeller (BET) method was utilized to calculate the specific surface areas by using the nonlocal density functional theory (NLDFT) model, and the pore volume was derived from 
the sorption curve. Thermogravimetric analysis from 30 to $700^{\circ} \mathrm{C}$ was carried out on a Mettler Toledo thermogravimetric analyzer in an air atmosphere using a $3^{\circ} \mathrm{C} / \mathrm{min}$ ramp time. Powder X-ray diffraction (PXRD) data were recorded on a Bruker DiscoverD8 model diffractometer by depositing powder on plastic substrate, from $2 \theta=1^{\circ}$ up to $30^{\circ}$ with $0.05^{\circ}$ increment.

\section{Synthesis of PyP}

meso-Tetra(4-aminophenyl)porphine Cu(II) (0.2 g, $0.27 \mathrm{mmol})$ and naphthalenetetracarboxylic dianhydride $(0.15 \mathrm{~g}$, $0.54 \mathrm{mmol}$ ) were added to a dry two-neck flask containing a magnetic stir bar fitted to a reflux condenser. The mixture was stirred at $0^{\circ} \mathrm{C}$ in $20 \mathrm{~mL} m$-cresol under $\mathrm{N}_{2}$ flow until all the solids dissolved. The mixture was heated to r.t, and stirred at this temperature overnight. $5 \mathrm{~mL}$ of isoquinoline was added and stirred at $80^{\circ} \mathrm{C}$ for four hours, $180^{\circ} \mathrm{C}$ for $16 \mathrm{~h}$. The reaction mixture was cooled down, and the precipitate was isolated from the solution by filtration, washed with DMF. Then the solid was grounded into a fine powder, which was subsequently washed copiously with methanol/acetone to give a purple powder. Yield: 64\%. Elemental Analysis (\%) calculated for PyP, theory: C (72.03), H (2.69), N (9.33), found C (71.49), H (2.12), N (10.64), respectively.

\section{Results and Discussion}

Fourier transform infrared spectroscopy (FT-IR) shows the disappearance of the $\mathrm{N}-\mathrm{H}$ stretching frequencies of the mesotetraphenylamino porphyrin. The PyP polymer exhibited a strong stretching frequency at $995 \mathrm{~cm}^{-1}$ which is characteristic of the $\mathrm{N}-\mathrm{Cu}$ vibration band which remained unchanged in the corresponding polymers and also showed a $60-70 \mathrm{~cm}^{-1}$ shift and a broadened peak in the carbonyl region of the polymer, corresponding to the naphthalenetetracarboxylic dianhydride (Figure 1). The chemical linkages for polyimides were confirmed by the characteristic bands at 1780 and $1716 \mathrm{~cm}^{-1}$ due to the symmetric and asymmetric vibrations of the $\mathrm{C}=\mathrm{O}$ group, and broad stretch at $1349 \mathrm{~cm}^{-1}$ corresponds to the stretching vibration of the $\mathrm{C}-\mathrm{N}-\mathrm{C}$ linkages. Also, the absorption bands for amino and anhydride were absent, indicating that the reactive groups have been completely polymerized.

The UV-Vis diffuse reflectance spectra (Figure 2) showed broad peaks between 200 and $800 \mathrm{~nm}$ with maximum at $420 \mathrm{~nm}$, which is a characteristic for porphyrins, whereas three separated bands with maxima at $560 \mathrm{~nm}, 590$, and $620 \mathrm{~nm}$ were observed. The presence of porphyrin units in the PyP was confirmed by the appearance of broad Soret (420$454 \mathrm{~nm}$ ) and Q bands (560, 590, and $620 \mathrm{~nm}$ ). The solidstate ${ }^{13} \mathrm{C}$ cross polarization and magic angle spinning (CPMAS) NMR spectra (Figure 3) also support the presence of porphyrin monomers in the polymer framework. The NMR spectrum exhibited a signal at $\delta=151$ ppm which corresponds to the $\mathrm{N}-\mathrm{C}=\mathrm{O}$ imide bonds, which was not present in the NMR spectrum of meso-tetraphenylamino porphyrin. Other peaks at $\delta=131.3$ and $93.7 \mathrm{ppm}$ were assigned to

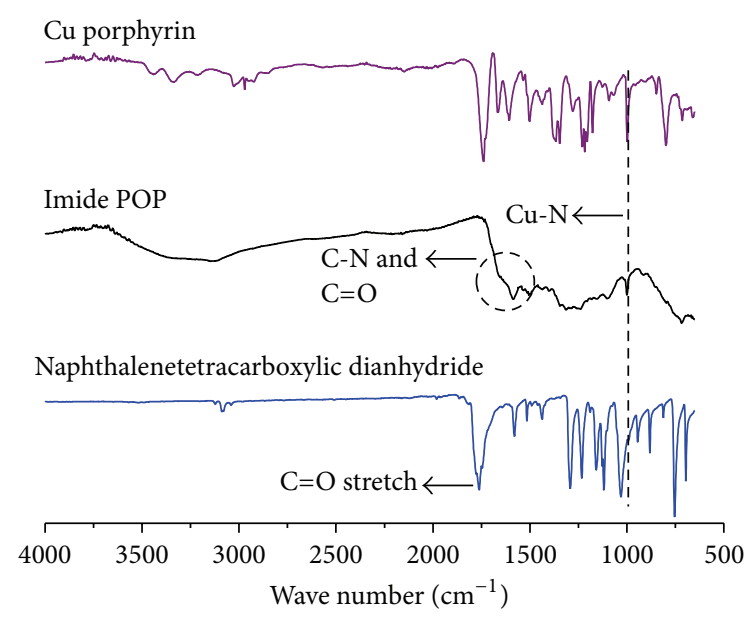

FIGURE 1: IR spectra of PyP, meso-tetraphenylamino porphyrin, and naphthalenetetracarboxylic dianhydride.

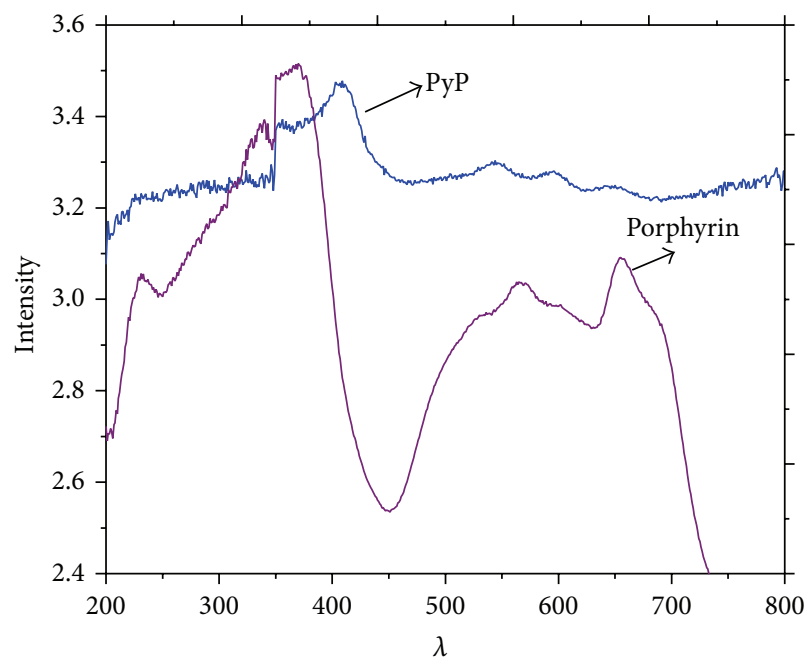

Figure 2: Solid-state absorption spectra PyP (blue), 1 (purple) as powders using a praying mantis diffuse reflectance accessory.

the aromatic carbon atoms of the PyP by comparison with the spectrum of the porphyrin. ${ }^{13} \mathrm{C} \mathrm{CP}$-MAS spectra of the $\mathrm{PyP}$ and porphyrin display similar chemical shifts to other reported polyimide frameworks and porphyrins $[15,16,18-$ 20].

Powder X-ray diffraction ( $\mathrm{Cu} \mathrm{K} \alpha$ radiation) was used to examine the crystallinity of the as-synthesized PyP. As expected, the diffraction pattern did not display any diffraction peaks indicating the amorphous nature of PyP. The scanning electron microscopy (SEM) image revealed a flakelike morphology of randomly aggregated particles of the PyP powder (Figure 4). To measure the thermal stability of the PyP, the sample was placed in the thermogravimetric analyzer under a flow of air (Figure 5). The TGA curve is similar to those of other reported imide/imine linked porous organic polymers, loosing $10 \%$ of the weight at $370^{\circ} \mathrm{C}$ and gradually decomposing at $500^{\circ} \mathrm{C}$ [16]. 


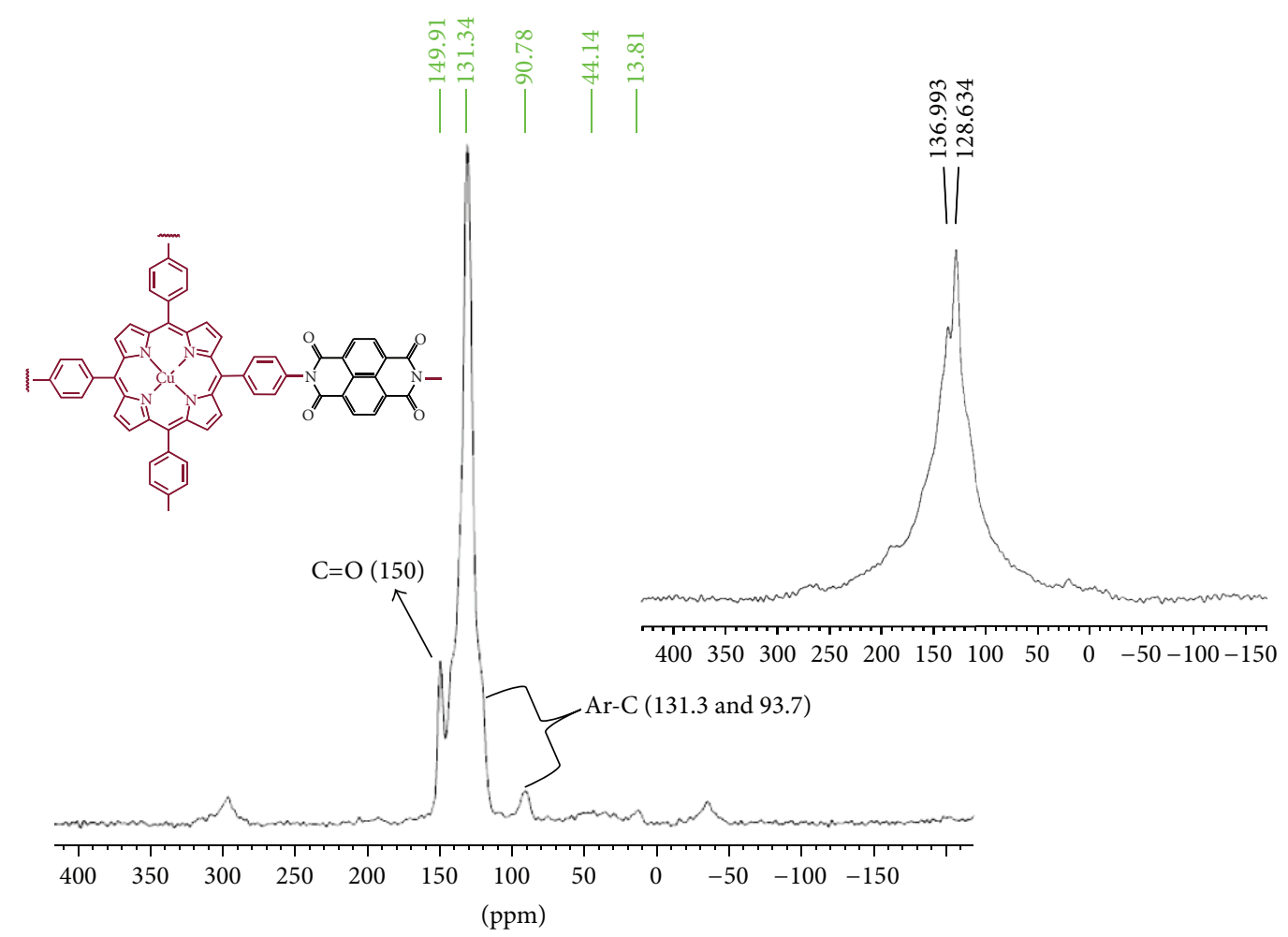

Figure 3: Solid-state ${ }^{13} \mathrm{C}$ CP-MAS NMR spectrum of PyP recorded at MAS rate of $10 \mathrm{kHz}$.

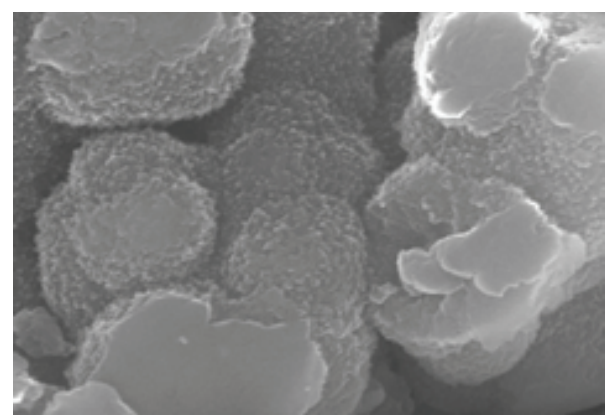

FIgURE 4: Scanning electron microscope image of the PyP.

The porous nature of the PyP was further evaluated by $\mathrm{N}_{2}$ and $\mathrm{H}_{2}$ uptake measurements at $77 \mathrm{~K} / 1$ bar and $\mathrm{CH}_{4}$ and $\mathrm{CO}_{2}$ at $273 \mathrm{~K}, 298 \mathrm{~K} / 1$ bar. The fully reversible $\mathrm{N}_{2}$ isotherm depicted in Figure 6(a) shows a rapid uptake at low pressure ( $0-0.1$ bar) indicating a permanent microporous nature and a gradual increase at higher pressures (0.1-1 bar). A surface area of $423 \mathrm{~m}^{2} \mathrm{~g}^{-1}$ was obtained by applying the BET (Brunauer-Emmett-Teller) model within the pressure range of $0-1$ bar. These values are comparable to those of triphenylamine-based polyimide frameworks [15, 16, 18-20]. The effect of framework interpenetration on the porous nature of the PyP was observable from pore size distribution using nonlocal density functional theory (NLDFT). Pore size distribution was estimated by fitting $\mathrm{N}_{2}$ uptake isotherms using NLDFT and found two different pore sizes around $6 \AA$ and $11 \AA$ (Figure 7) and the pore volume from single

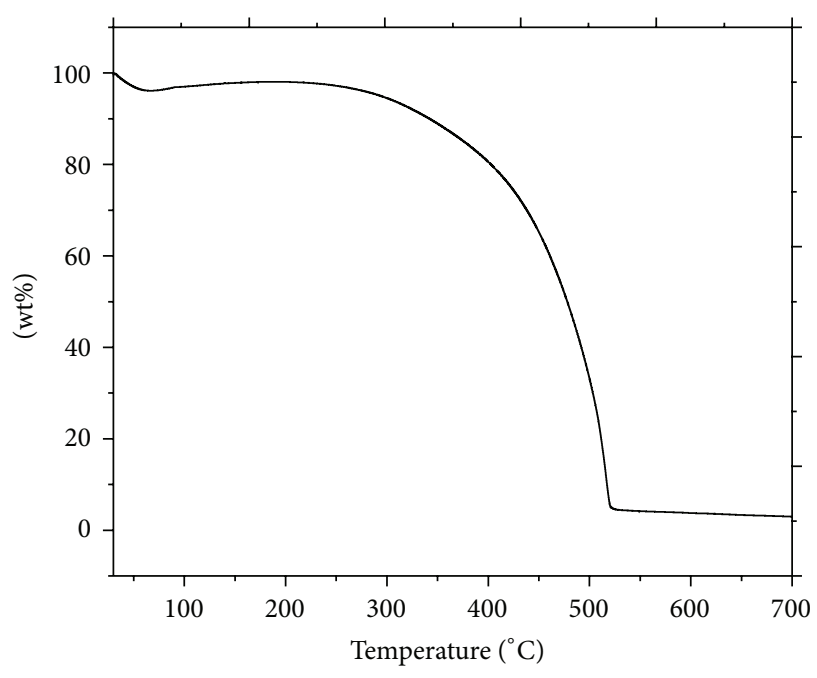

FIgure 5: TGA of PyP obtained at up to $700^{\circ} \mathrm{C}$ using a linear $3^{\circ} \mathrm{C} / \mathrm{min}$ ramp method.

point measurements was calculated to be $0.1 \mathrm{~cm}^{3} \mathrm{~g}^{-1}\left(P / P_{o}=\right.$ $0.1)$. We believe that the two different pore sizes are due to interpenetration of the frameworks. As we stated earlier, crystalline and amorphous polymers containing nitrogen functionalized pore walls have shown enhanced $\mathrm{CO}_{2}$ uptakes and selectivity. To investigate the impact of these parameters on the uptake of small gases such as $\mathrm{H}_{2}, \mathrm{CH}_{4}$, and $\mathrm{CO}_{2}$ and the selective $\mathrm{CO}_{2}$ adsorption over $\mathrm{CH}_{4}$, we measured isotherms (Figure 6) and calculated their isosteric enthalpies 


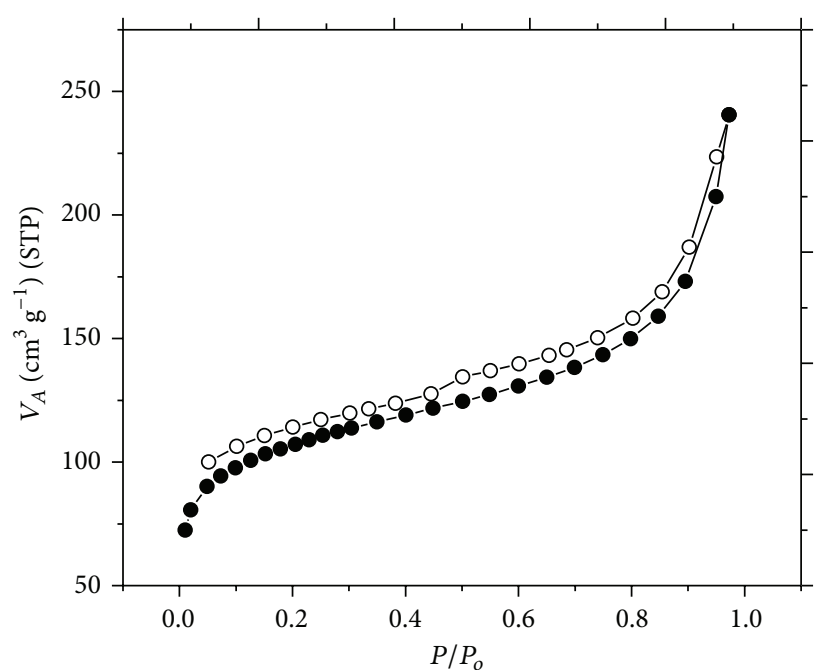

(a)

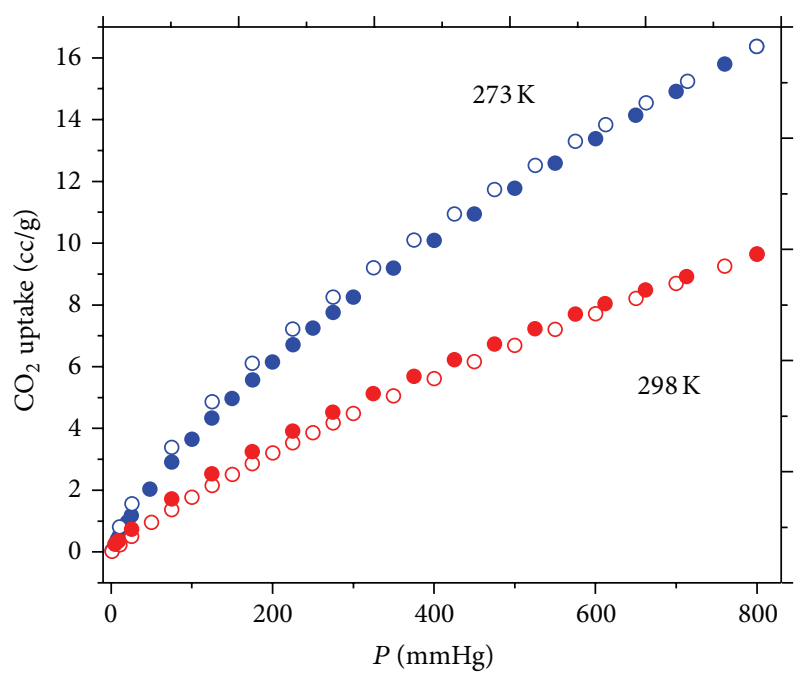

(c)

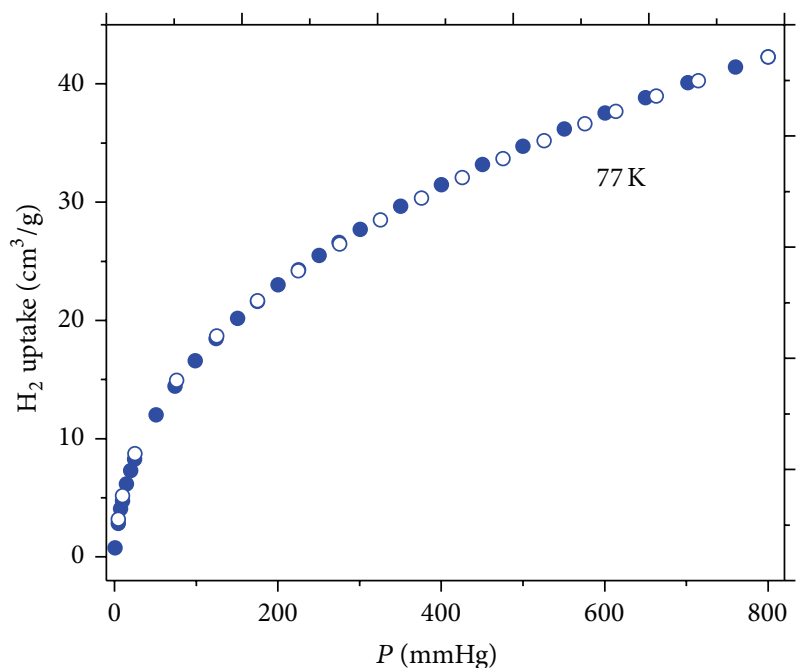

(b)

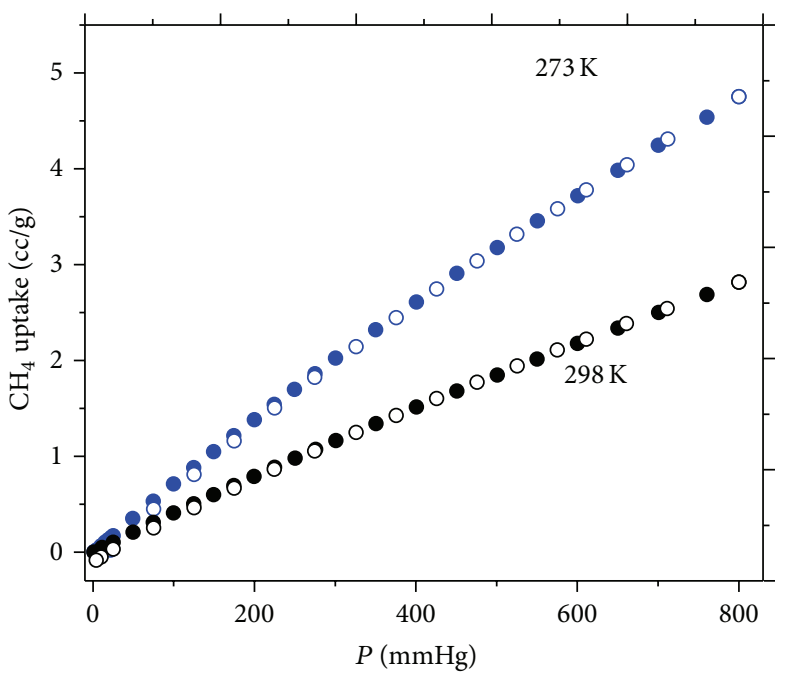

(d)

Figure 6: (a) $\mathrm{N}_{2}$ at $77 \mathrm{~K}$, (b) $\mathrm{H}_{2}$ at $77 \mathrm{~K}$, (c) $\mathrm{CO}_{2}$ at $273 \mathrm{~K}$ and $298 \mathrm{~K}$, and (d) $\mathrm{CH}_{4}$ at $273 \mathrm{~K}$ and $298 \mathrm{~K}$. Adsorption (filled symbols) and desorption (empty symbols) isotherm curves.

of adsorption $\left(\mathrm{Q}_{\mathrm{st}}\right)$ from Henry's constants (Figure 8) and compared these values to those of recently reported nitrogen containing porous organic polymers. $\mathrm{H}_{2}, \mathrm{CO}_{2}$, and $\mathrm{CH}_{4}$ isotherms are fully reversible and exhibit a gradual rise at low pressures. The $\mathrm{PyP}$ showed a moderate $\mathrm{CO}_{2}$ uptake of $3.4 \mathrm{wt} \%$ at $273 \mathrm{~K} / 1$ bar which is lower than that of triphenylene-based imide networks measured at $195 \mathrm{~K} / 1 \mathrm{bar}$ [10]. To determine the binding affinity of PyP for $\mathrm{CO}_{2}$, we calculated $\mathrm{Q}_{\mathrm{st}}$ using the Van't Hoff equation $\left(19.2 \mathrm{~kJ} \mathrm{~mol}^{-1}\right)$. These values are slightly lower than those reported for organic polymers in general but lower than those of nitrogen-rich MOFs. The readily reversible sorption/desorption behavior and moderate $\mathrm{Q}_{\mathrm{st}}$ indicate that $\mathrm{CO}_{2}$ interactions with pore walls are weak enough to allow for material regeneration without applying heat. We have also measured $\mathrm{H}_{2}$ and $\mathrm{CH}_{4}$ storage properties of $\mathrm{PyP} . \mathrm{H}_{2}$ uptake was only $0.4 \mathrm{wt} \%$ at $77 \mathrm{~K}$ and 1 bar (Figure 6(b)), which is lower than that for other microporous organic polymers. Similarly, the measured $\mathrm{CH}_{4}$ uptake was $0.32 \mathrm{wt} \%$ at $273 \mathrm{~K}$ and 1 bar (Figure $6(\mathrm{~d})$ ). Both isotherms are completely reversible and exhibit a gradual rise at low pressure and reach maxima of $14-27 \mathrm{mg} \mathrm{g}^{-1}$ at $273 \mathrm{~K}$ and $9-19 \mathrm{mg} \mathrm{g}^{-1}$ at $298 \mathrm{~K} . \mathrm{Q}_{\mathrm{st}}$ for $\mathrm{CH}_{4}$ was calculated by using adsorption data collected at $273 \mathrm{~K}$. At zero coverage, $\mathrm{Q}_{\mathrm{st}}$ is $13.9 \mathrm{~kJ} \mathrm{~mol}^{-1}$. Based on these values we compared $\mathrm{CO}_{2}$ uptake and selectivity over $\mathrm{CH}_{4}$ to evaluate the potential use of PyP for selective $\mathrm{CO}_{2}$ capture. At $273 \mathrm{~K}$ and 1 bar, $\mathrm{CO}_{2}$ uptake is $3.4 \mathrm{wt} \%$, whereas that of $\mathrm{CH}_{4}$ is only $0.32 \mathrm{wt} \%$. This preferential higher affinity for $\mathrm{CO}_{2}$ could be potentially useful for $\mathrm{CO}_{2}$ capture. We calculated the gas adsorption selectivities from the initial slope ratios estimated from Henry's constants. 


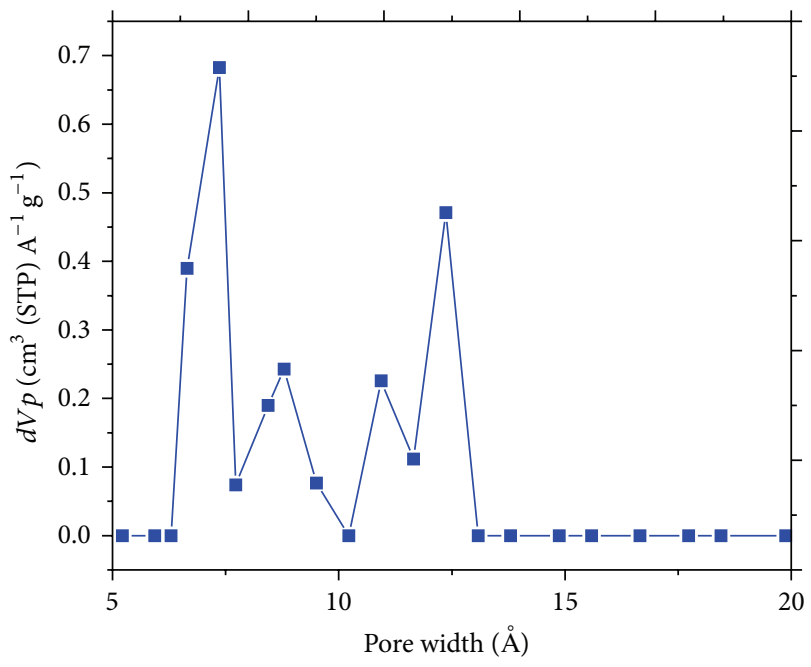

(a)

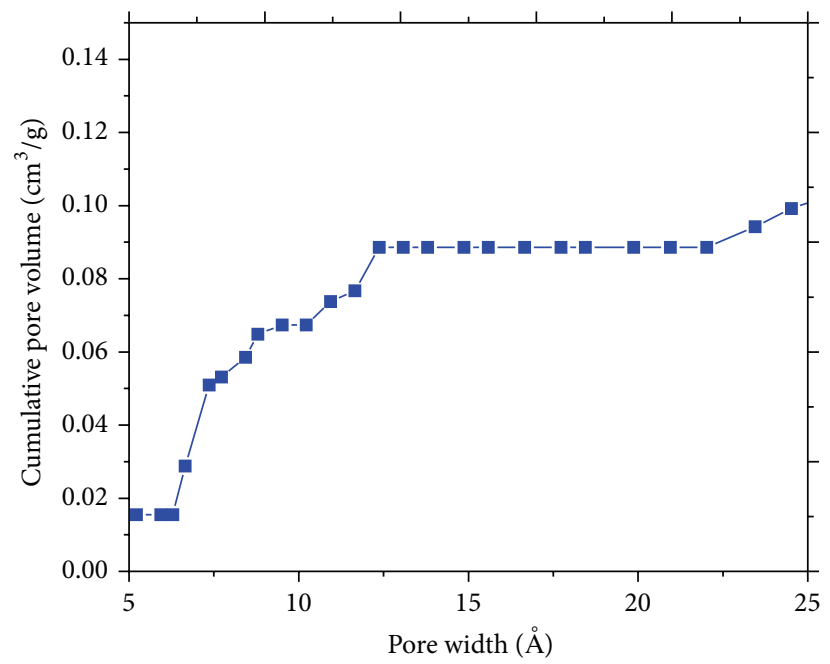

(b)

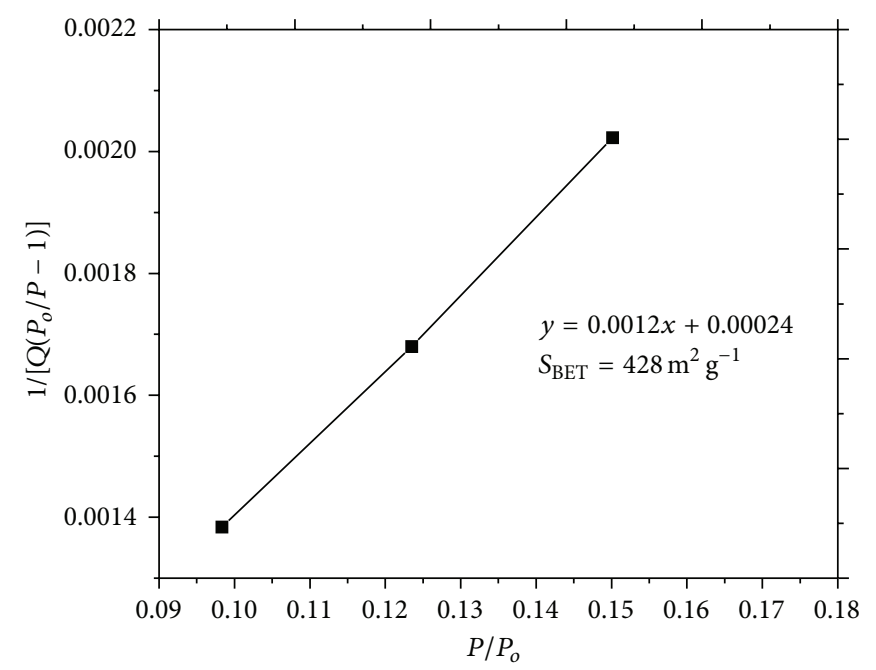

(c)

FIgURE 7: Differential (a) and cumulative (b) pore size distribution plot of PyP from the application of the NLDFT model to $\mathrm{N}_{2}$ isotherm. BET plot (c) for PyPcalculated from isotherm data.

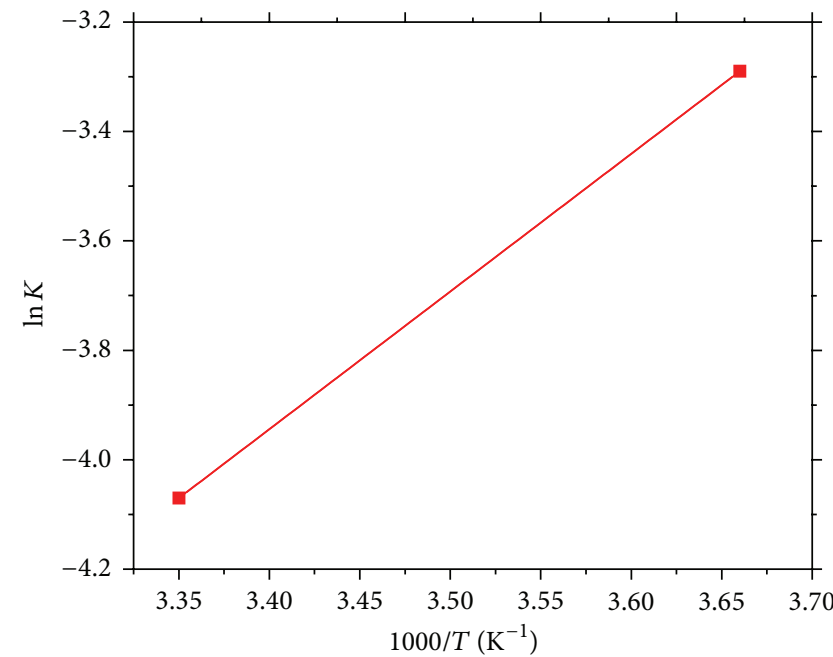

(a)

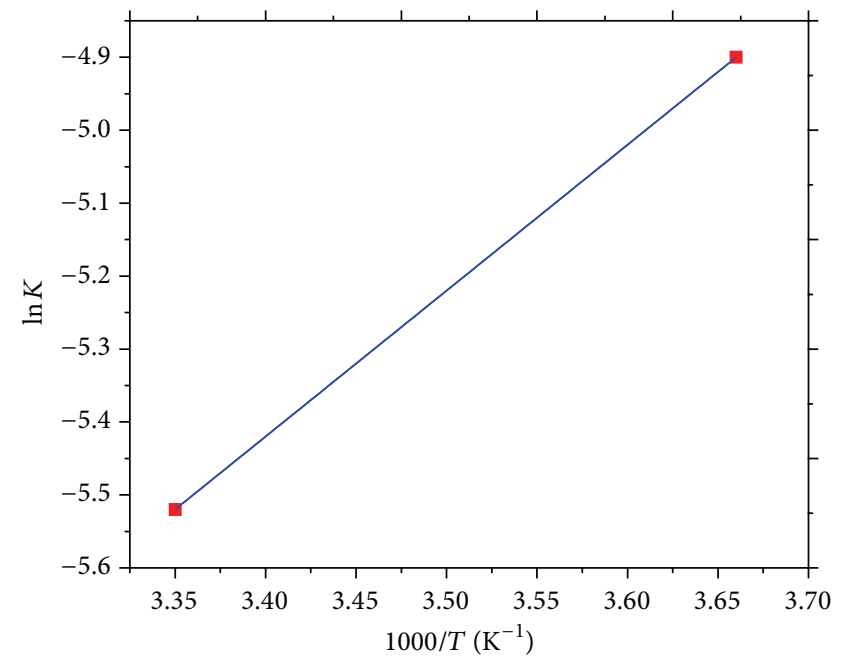

(b)

FIGURE 8: Van't Hoff plots of $\mathrm{CO}_{2}$ (red) and $\mathrm{CH}_{4}$ (blue). 


\section{Calculation of Isosteric Heat of Adsorption}

The adsorption enthalpy at zero coverage was calculated from Henry constant using the Van't Hoff equation as

$$
\ln K=-\frac{\Delta H}{R T}+\frac{\Delta S}{R} .
$$

$K$ is Henry constant and $T$ is temperature, plotting $\ln K$ versus $1000 / T$.

\section{Conclusions}

In conclusion, we have synthesized, characterized, and described the use of an imide linked porphyrin-based porous polymer framework, $\mathrm{PyP}$, for $\mathrm{CO}_{2}$ capture. In addition to the promising selective $\mathrm{CO}_{2}$ capture properties, this $\mathrm{PyP}$ also showed some storage capabilities for $\mathrm{H}_{2}$ at $77 \mathrm{~K}$ and for $\mathrm{CH}_{4}$ at $273 \mathrm{~K}$.

\section{Conflict of Interests}

The authors declare that there is no conflict of interests regarding the publication of this paper.

\section{Acknowledgments}

This work was generously supported by NSF Grant DMR1205302 (PREM Program) and the Robert A. Welch Foundation, Grant no. AH-0033.

\section{References}

[1] C. Heo and J.-H. Chang, "Polyimide nanocomposites based on functionalized graphene sheets: morphologies, thermal properties, and electrical and thermal conductivities," Solid State Sciences, vol. 24, pp. 6-14, 2013.

[2] A. P. Côté, A. I. Benin, N. W. Ockwig, M. O’Keeffe, A. J. Matzger, and O. M. Yaghi, "Chemistry: porous, crystalline, covalent organic frameworks," Science, vol. 310, no. 5751, pp. 1166-1170, 2005.

[3] J. W. Colson and W. R. Dichtel, "Rationally synthesized twodimensional polymers," Nature Chemistry, vol. 5, no. 6, pp. 453465, 2013.

[4] X. Feng, X. Ding, and D. Jiang, "Covalent organic frameworks," Chemical Society Reviews, vol. 41, no. 18, pp. 6010-6022, 2012.

[5] K. Sumida, D. L. Rogow, J. A. Mason et al., "Carbon dioxide capture in metal-organic frameworks," Chemical Reviews, vol. 112, no. 2, pp. 724-781, 2012.

[6] Y. Xu, S. Jin, H. Xu, A. Nagai, and D. Jiang, "Conjugated microporous polymers: Design, synthesis and application," Chemical Society Reviews, vol. 42, no. 20, pp. 8012-8031, 2013.

[7] M. Hashem, C. G. Bezzu, B. M. Kariuki, and N. B. McKeown, "Enhancing the rigidity of a network polymer of intrinsic microporosity by the combined use of phthalocyanine and triptycene components," Polymer Chemistry, vol. 2, no. 10, pp. 2190-2192, 2011.

[8] N. B. McKeown, "Nanoporous molecular crystals," Journal of Materials Chemistry, vol. 20, no. 47, pp. 10588-10597, 2010.
[9] V. S. P. K. Neti, X. Wu, S. Deng, and L. Echegoyen, "Selective $\mathrm{CO}_{2}$ capture in an imine linked porphyrin porous polymer," Polymer Chemistry, vol. 4, no. 17, pp. 4566-4569, 2013.

[10] V. S. P. K. Neti, X. Wu, S. Deng, and L. Echegoyen, "Synthesis of a phthalocyanine and porphyrin $2 \mathrm{D}$ covalent organic framework," CrystEngComm, vol. 15, no. 35, pp. 6892-6895, 2013.

[11] V. S. P. K. Neti, X. Wu, M. Hosseini, R. A. Bernal, S. Deng, and L. Echegoyen, "Synthesis of a phthalocyanine 2D covalent organic framework," CrystEngComm, vol. 15, no. 36, pp. 7157-7160, 2013.

[12] V. S. P. K. Neti, X. Wu, P. Peng, S. Deng, and L. Echegoyen, "Synthesis of a benzothiazole nanoporous polymer for selective $\mathrm{CO}_{2}$ adsorption," RSC Advances, vol. 4, no. 19, pp. 9669-9672, 2014.

[13] A. Phan, C. J. Doonan, F. J. Uribe-Romo, C. B. Knobler, M. Okeeffe, and O. M. Yaghi, "Synthesis, structure, and carbon dioxide capture properties of zeolitic imidazolate frameworks," Accounts of Chemical Research, vol. 43, no. 1, pp. 58-67, 2010.

[14] P. Pandey, O. K. Farha, A. M. Spokoyny et al., 'A 'click-based' porous organic polymer from tetrahedral building blocks," Journal of Materials Chemistry, vol. 21, no. 6, pp. 1700-1703, 2011.

[15] M. G. Rabbani and H. M. El-Kaderi, “Template-free synthesis of a highly porous benzimidazole-linked polymer for $\mathrm{CO}_{2}$ capture and $\mathrm{H}_{2}$ storage," Chemistry of Materials, vol. 23, no. 7, pp. 16501653, 2011.

[16] O. K. Farha, A. M. Spokoyny, B. G. Hauser et al., "Synthesis, properties, and gas separation studies of a robust diimide-based microporous organic polymer," Chemistry of Materials, vol. 21, no. 14, pp. 3033-3035, 2009.

[17] S. Yang, J. Sun, A. J. Ramirez-Cuesta et al., "Selectivity and direct visualization of carbon dioxide and sulfur dioxide in a decorated porous host," Nature Chemistry, vol. 4, no. 11, pp. 887894, 2012.

[18] A. M. Shultz, O. K. Farha, J. T. Hupp, and S. T. Nguyen, "Synthesis of catalytically active porous organic polymers from metalloporphyrin building blocks," Chemical Science, vol. 2, no. 4, pp. 686-689, 2011.

[19] K. V. Rao, R. Haldar, C. Kulkarni, T. K. Maji, and S. J. George, "Perylene based porous polyimides: tunable, high surface area with tetrahedral and pyramidal monomers," Chemistry of Materials, vol. 24, no. 6, pp. 969-971, 2012.

[20] V. S. P. K. Neti, J. Wang, S. Deng, and L. Echegoyen, "Selective $\mathrm{CO}_{2}$ adsorption in a porphyrin polymer with benzimidazole linkages," RSC Advances, vol. 5, pp. 10960-10963, 2015. 

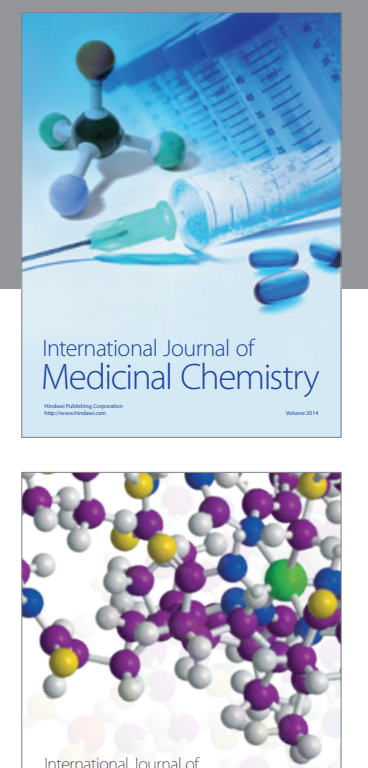

\section{Carbohydrate} Chemistry

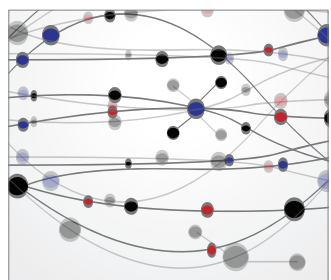

The Scientific World Journal
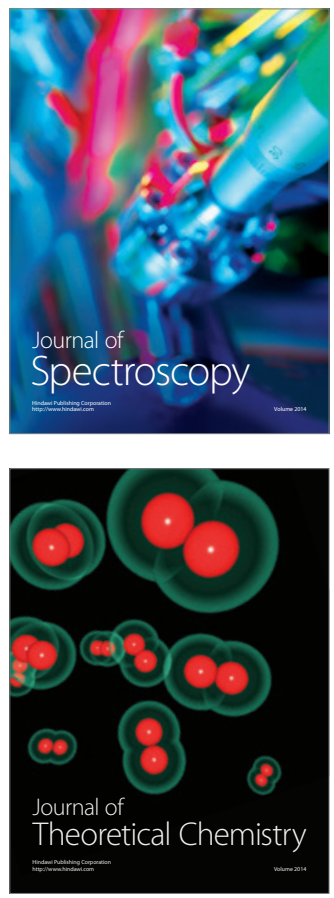
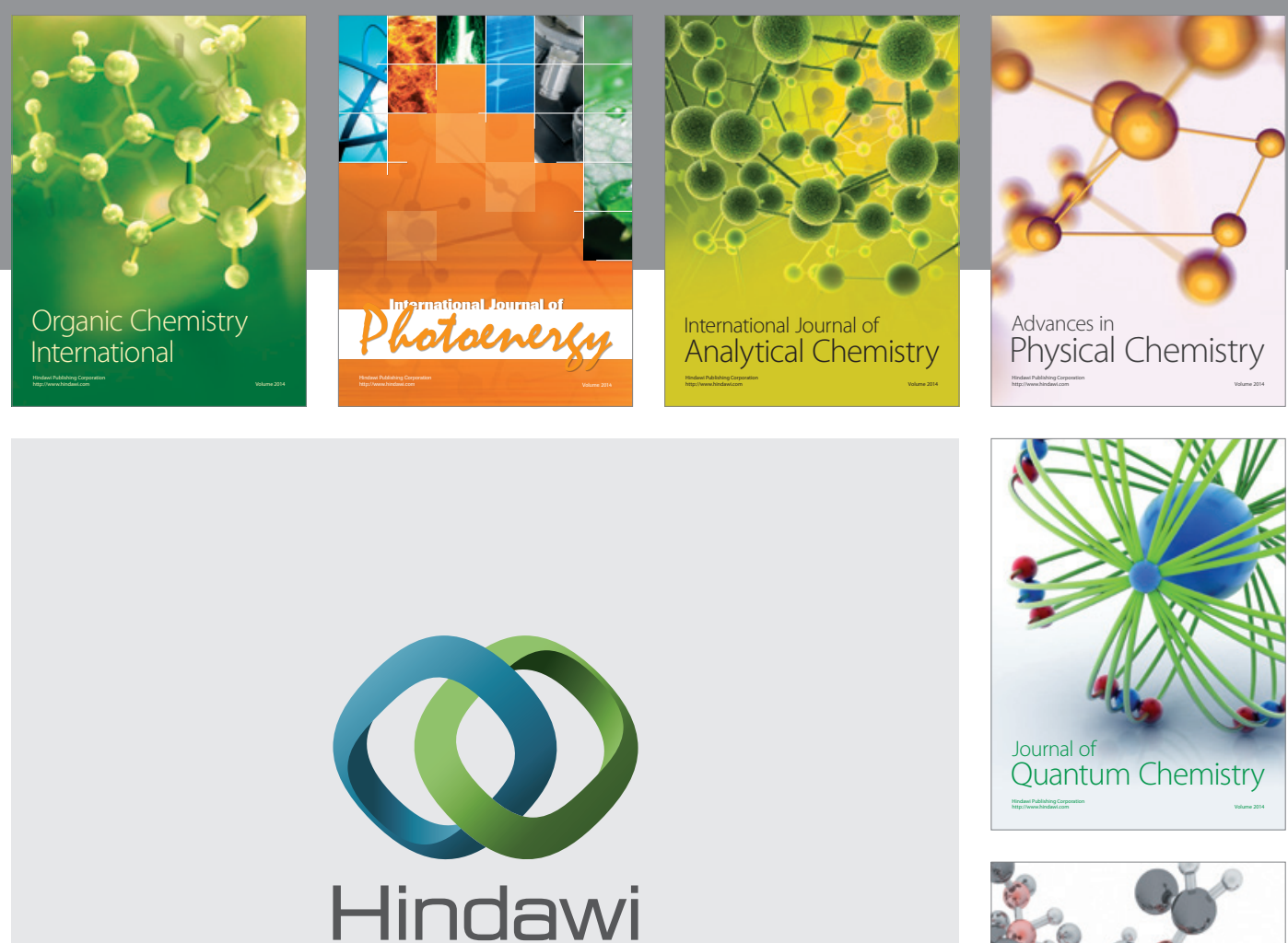

Submit your manuscripts at

http://www.hindawi.com

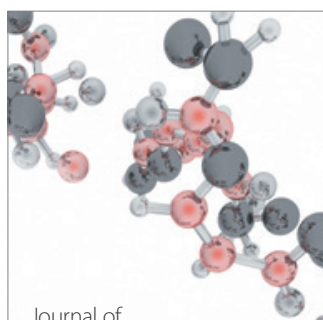

Analytical Methods

in Chemistry

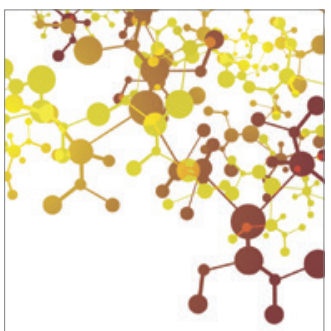

Journal of

Applied Chemistry

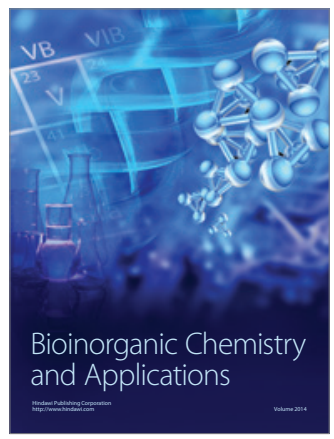

Inorganic Chemistry
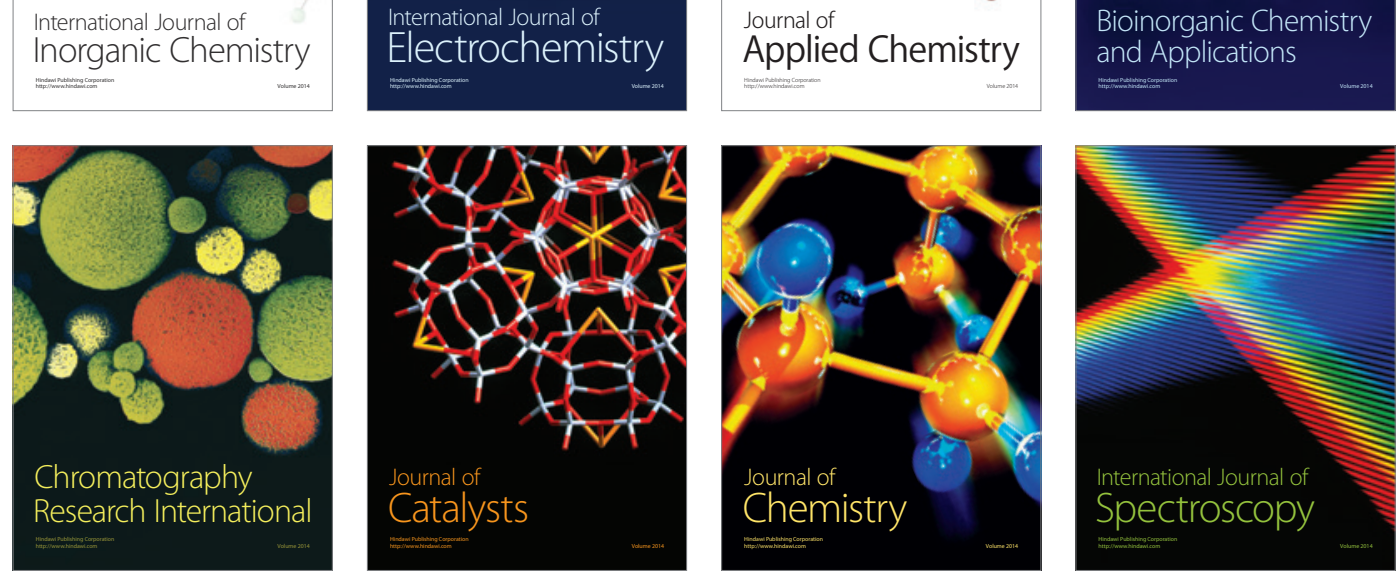